\title{
LTR retrotransposons and the evolution of dosage compensation in Drosophila
}

\author{
Lilya V Matyunina, Nathan J Bowen and John F McDonald*
}

Address: School of Biology and Petit Institute for Bioengineering and Bioscience, Georgia Institute of Technology, Atlanta, GA 30332, USA

Email: Lilya V Matyunina - lilya.matyunina@biology.gatech.edu; Nathan J Bowen - nathan.bowen@biology.gatech.edu;

John F McDonald* - john.mcdonald@biology.gatech.edu

* Corresponding author

Published: 4 June 2008

BMC Molecular Biology 2008, 9:55 doi:10.1 186/147/-2199-9-55

This article is available from: http://www.biomedcentral.com/I47/-2/99/9/55

(C) 2008 Matyunina et al; licensee BioMed Central Ltd.

This is an Open Access article distributed under the terms of the Creative Commons Attribution License (http://creativecommons.org/licenses/by/2.0), which permits unrestricted use, distribution, and reproduction in any medium, provided the original work is properly cited.
Received: 9 May 2008

Accepted: 4 June 2008

\begin{abstract}
Background: Dosage compensation in Drosophila is the epigenetic process by which the expression of genes located on the single $X$-chromosome of males is elevated to equal the expression of $X$-linked genes in females where there are two copies of the $\mathrm{X}$-chromosome. While epigenetic mechanisms are hypothesized to have evolved originally to silence transposable elements, a connection between transposable elements and the evolution of dosage compensation has yet to be demonstrated.

Results: We show that transcription of the Drosophila melanogaster copia LTR (long terminal repeat) retrotransposon is significantly down regulated when in the hemizygous state. DNA digestion and chromatin immunoprecipitation (ChIP) analyses demonstrate that this down regulation is associated with changes in chromatin structure mediated by the histone acetyltransferase, MOF. MOF has previously been shown to play a central role in the Drosophila dosage compensation complex by binding to the hemizygous $\mathrm{X}$-chromosome in males.

Conclusion: Our results are consistent with the hypothesis that MOF originally functioned to silence retrotransposons and, over evolutionary time, was co-opted to play an essential role in dosage compensation in Drosophila.
\end{abstract}

\section{Background}

Retrotransposons are a major component of the genomes of higher eukaryotes and have been identified as a significant source of loss-of-function and regulatory mutations [1]. Over evolutionary time host genomes have developed mechanisms to mitigate the mutational potential of retrotransposons by transcriptionally silencing or otherwise blocking their transpositional activity [2]. One of the primary mechanisms by which retrotransposons are transcriptionally silenced is by methylation and/or other epigenetic mechanisms. Indeed it has been hypothesized that most, if not all, epigenetic mechanisms originally evolved as a defense against retrotransposons and have subsequently been co-opted for other essential cellular functions $[3,4]$.

Approximately $10 \%$ of the Drosophila melanogaster genome is comprised of retrotransposons, the majority of which are LTR retrotransposons. LTR retrotransposon insertions are a major source of mutations in D. melanogaster and are believed to have contributed significantly to genome evolution [5]. While histone acetylation and other epigenetic mechanisms are believed to play an essential role in dosage compensation and other vital 
functions in D. melanogaster, little is known about the role of these mechanisms in the regulation of retrotransposons in this species [e.g., [6-8]]. In this paper, we present genetic and molecular evidence that the histone acetyltransferase, MOF, is involved in the transcriptional repression of the copia and perhaps other families of LTR retrotransposons in Drosophila. Our findings are consistent with the hypothesis that MOF may have originally functioned to silence retrotransposons and was subsequently co-opted for its role in dosage compensation.

\section{Results}

A series of Drosophila melanogaster strains transformed with a copia LTR-CAT (chloramphenicol acetyltransferase) reporter construct (Figure 1) was monitored for CAT activity in larvae and adult flies carrying the construct in the hemizygous vs. the homozygous state. Inconsistent with a simple additive model, CAT activity was found, on average, to be $\sim 7$-fold higher in strains homozygous vs. hemizygous for the construct (Table 1). In contrast, CAT activity of strains in which two copies of the hemizygous construct were present at non-homologous locations was consistent with an additive model (Table 2 and 3). Thus, the observed non-additive effect between hemizygous and homozygous copia elements is cis-dependent.

Previous studies in other experimental systems have demonstrated that transposable elements are often partially or totally repressed due to host-mediated modifications in chromatin structure [e.g., [9-11]]. To determine if the non-additive effect we observed in copia expression is also associated with alterations in chromatin structure, we tested the ability of the ApaI restriction enzyme to access and digest a site in the copia LTR-CAT construct in larval chromatin extracts [12]. The results indicate that hemizygous copies of the copia LTR-CAT construct are

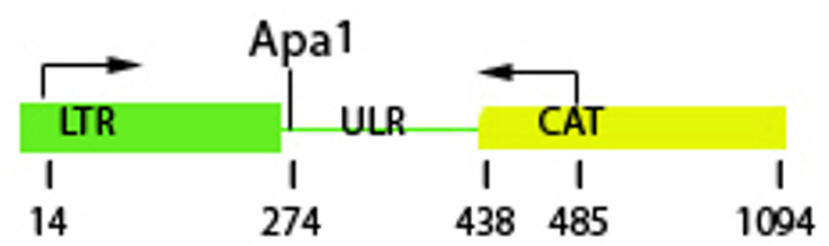

\section{Figure I}

Structure of the copia LTR-CAT construct. Position of Apa I site (274 bp) and the 5' (I4 bp) and 3' (485 bp) PCR primer binding sites are shown (LTR $=5^{\prime}$ copia long terminal repeat; ULR= copia untranslated leader region; CAT $=$ bacterial chloramphenicol acetyltransferase reporter gene (see Methods for primer sequences). being down regulated due, at least in part, to a chromatin mediated effect (Figure 2).

A number of genes have been shown to regulate chromatin structure in Drosophila [e.g., $[6,7,13,14]]$. To determine if members of this class of genes are involved in repressing the expression of hemizygous copies of the copia LTR-CAT construct, we monitored CAT activity in larvae or adult flies hemizygous for the construct within a series of genetic backgrounds mutant for genes known to affect chromatin structure in Drosophila $[13,14][\mathrm{LOW} /+, \mathrm{HDAC}$ 1326/+, HDAC 1328/+, E(z) $28 /+, E(z)^{61 /+, P s c 25 /+, ~ S x l f l} /+$, and $m^{1} f^{1}$ (Table 4, see Additional files 12, Tables 1, 2, 3, 4 ), presence of extra Y chromosome or absence of $Y$ chromosome (see Additional file 2, Table 5)]. With the exception of $m^{\prime} f^{1}$, each of the alleles tested display a dominant mutant phenotype in heterozygotes and are (embryonic) lethal when homozygous. Males hemizygous for the mutant (X-linked) mof ${ }^{1}$ allele develop to the $3^{\text {rd }}$ instar stage but fail to metamorphose and hatch. In contrast, the phenotype and viability of females homozygous for the mutant mof $^{1}$ allele is unaffected.

No significant effect on LTR-CAT expression was detected in genetic backgrounds heterozygous for the dominant mutant LOW, E(z)61 and Sxl fl alleles (Table 4; see Additional file 2, Tables 1,3 and 4). Flies heterozygous for the dominant mutant HDAC 1326, HDAC 1328, E(z)28 and Psc25 alleles and for the presence of an extra Y chromosome or absence of a Y chromosome displayed a slight but significant $(\mathrm{p}<0.01)$ decrease in CAT activity relative to controls (Table 4, see Additional file 2, Tables 2, 3 and 5). In contrast, a highly significant $(\mathrm{p}<0.001)$ increase in the expression of the copia LTR-CAT constructs was observed in male larvae mutant for the recessive (hemizygous) $\mathrm{X}$ linked mof 1 allele (males absent on first) (Table 5). This increase in expression in the mutant mof 1 background was observed in all of the independent copia LTR-CAT transformants examined (Table 5). Thus, the effect is not dependent upon the chromosomal location of the construct.

The protein product of the mof gene (MOF) is a member of the MYST family of histone acetyltransferases and, as part of the Drosophila MSL (male specific lethal) complex $[15,16]$, has been shown to play an essential role in dosage compensation [e.g., [17-19]]. Our results are consistent with the effect of mof on copia LTR-CAT expression being chromatin-mediated (Figure 2).

Since most active transposable elements (including LTR retrotransposons) are considered to be hemizygous in wild collected populations, we tested the effect of the mutant mof ${ }^{1}$ allele on global expression levels of copia and other families of LTR retrotransposons in a strain estab- 
Table I: Expression level of a copia LTR-CAT construct in nine stably transformed lines of Drosophila melanogaster made hemi- or homozygous for the construct

\begin{tabular}{|c|c|c|c|c|c|c|}
\hline Transformant & $\begin{array}{l}\text { Insert Chrom- } \\
\text { osomal Location }\end{array}$ & -------------- & CAT ACTIVITY & ------------ & --------------- & $\begin{array}{l}\text { RATIO (homo/ } \\
\text { hemi) }\end{array}$ \\
\hline & & $\begin{array}{c}\text { Female } \\
\text { homozygous }\end{array}$ & $\begin{array}{c}\text { Female } \\
\text { hemizygous }\end{array}$ & $\begin{array}{c}\text { Male } \\
\text { homozygous }\end{array}$ & $\begin{array}{c}\text { Male } \\
\text { hemizygous }\end{array}$ & \\
\hline $8-1$ & $2 \mathrm{~L} 40 \mathrm{E}$ & lethal & $3.03(0.33)$ & & & NA \\
\hline$"$ & & & & & lethal & $2.9(0.37)$ \\
\hline $9-1$ & $2 \mathrm{~L} 39 \mathrm{~A}$ & lethal & $0.32(0.04)$ & & & \\
\hline " & & & & & lethal & $0.36(0.06)$ \\
\hline $27-1$ & $2 \mathrm{R} 46 \mathrm{E}$ & lethal & $0.12(0.03)$ & & & \\
\hline$"$ & & & & & lethal & $0.11(0.01)$ \\
\hline $9-3$ & $3 \mathrm{~L} 80 \mathrm{~A}$ & $3.82(0.39)$ & $1.50(0.28)$ & & & 2.55 \\
\hline " & & & & $4.01(0.29)$ & $0.98(0.21)$ & 4.09 \\
\hline $9-4$ & $2 \mathrm{R} 57 \mathrm{~B}$ & $2.27(0.27)$ & $0.22(0.03)$ & & & $|0.3|$ \\
\hline$"$ & & & & $2.42(0.22)$ & $0.21(0.05)$ & 11.52 \\
\hline $9-6$ & $3 L 75 C$ & $3.60(0.23)$ & $0.56(0.13)$ & & & 6.43 \\
\hline " & & & & $3.65(0.05)$ & $0.32(0.02)$ & $11.4 \mid$ \\
\hline \multirow{2}{*}{$14-2$} & $4102 B$ & $1.10(0.35)$ & $0.28(0.03)$ & & & 3.93 \\
\hline & & & & $1.26(0.09)$ & $0.26(0.02)$ & 4.85 \\
\hline \multirow[t]{2}{*}{ |4-| } & $\times 16 \mathrm{~B}$ & $3.91(0.10)$ & $0.91(0.09)$ & & & 4.3 \\
\hline & & & & NA & $3.29(0.04)$ & NA \\
\hline \multirow[t]{3}{*}{$39-2$} & $\times 5 \mathrm{~A}$ & $1.88(0.13)$ & $0.20(0.04)$ & & & 9.4 \\
\hline & & & & NA & $1.91(0.24)$ & NA \\
\hline & & & & & & Av: 6.88 \\
\hline
\end{tabular}

The results demonstrate a nearly seven-fold average increase in CAT activity in flies homozygous vs. hemizygous for the copia LTR-CAT construct

lished from a natural population (Athens, GA). The results of a series of RT-PCR and Northern analyses presented in Figure 3 demonstrate that males carrying the mutant mof ${ }^{1}$ allele display a consistent and significant increase in the expression of all families of LTR retrotransposons examined. This suggests that the association of mof with down regulated expression of hemizygous copies of copia may extend generally to other families of LTR retrotransposons in Drosophila.

While MOF is known to bind predominately to the male $\mathrm{X}$-chromosome, weak binding also occurs at multiple locations on autosomes of Drosophila melanogaster [19] (Figure 4a). We observed a substantially reduced level of autosomal binding of MOF in Drosophila simulans, a species closely related to melanogaster but known to have significantly fewer numbers of LTR retrotransposons (Figure 4b) [20]. This suggests that at least some of the autosomal binding of MOF may be to LTR retrotransposon sequences.
To directly determine if MOF can bind to copia elements, we performed chromatin immunoprecipitation (ChIP) analyses using the Drosophila melanogaster Kc167 (female) cell line and previously described Drosophila MOF, MSL-1 and MSL-3 antibodies [21]. In males, these three proteins combine with MSL-2 (not expressed in females) and are sequestered to the X-chromosome as part of the MSL complex [21]. In females, MOF, MSL-1 and MSL-3 have been shown to be associated with all chromosomes at a reduced but significant level [19]. Since copia elements are known to be located on the X-chromosome, we chose to conduct our ChIP assays with the female Kc167 cell line in order not to confound copia specific binding by MOF with the more generalized X-chromosome binding that occurs in males. Spt4 is an autosomal gene previously shown not to be subject to dosage compensation [22]. Rox is a well-established binding site for MOF within the context of the MSL complex [23]. Since it has been shown that MSL-1, MSL-3 and MOF bind with reduced affinity to the

Table 2: Expression level of copia LTR-CAT in hemizygous Drosophila melanogaster flies.

\begin{tabular}{|c|c|c|c|c|}
\hline Transformant & $9-3$ & $9-4$ & $9-6$ & $14-2$ \\
\hline Hemizygous $\sigma^{r}$ & $1.00 \pm 0.14$ & $0.32 \pm 0.04$ & $0.67 \pm 0.08$ & $0.22 \pm 0.01$ \\
\hline Hemizygous $q$ & $1.08 \pm 0.21$ & $0.32 \pm 0.02$ & $0.72 \pm 0.02$ & $0.31 \pm 0.07$ \\
\hline
\end{tabular}

CAT activity of transformed D. melanogaster strains carrying a single hemizygous LTR-CAT construct inserted at various locations. 
Table 3: Expression level of copia LTR-CAT in double hemizygous ( 2 hemizygous inserts in non-homologous chromosomal locations) of Drosophila melanogaster flies

\begin{tabular}{|c|c|c|c|c|c|}
\hline $\begin{array}{l}\text { Transformant } \\
\text { combination }\end{array}$ & $\begin{array}{l}\text { Insert chromosomal } \\
\text { locations }\end{array}$ & Male observed & Male expected & Female observed & Female expected \\
\hline $9-3 / 9-4$ & $3 \mathrm{~L} 80 \mathrm{~A} / 2 \mathrm{R}$ 57B & $1.32 \pm 0.12$ & 1.32 & $1.26 \pm 0.09$ & 1.41 \\
\hline $9-3 / 9-6$ & $3 \mathrm{~L} 80 \mathrm{~A} / 3 \mathrm{~L} 75 \mathrm{C}$ & $1.77 \pm 0.29$ & 1.67 & $1.44 \pm 0.19$ & 1.81 \\
\hline $9-3 / 14-2$ & $3 \mathrm{~L} 80 \mathrm{~A} / 4$ I02B & $1.31 \pm 0.18$ & 1.24 & $1.09 \pm 0.12$ & 1.78 \\
\hline $9-4 / 9-6$ & $2 \mathrm{R} 57 \mathrm{~B} / 3 \mathrm{~L} 75 \mathrm{C}$ & $1.03 \pm 0.21$ & 0.99 & $1.06 \pm 0.17$ & 1.04 \\
\hline $9-4 / 14-2$ & $2 \mathrm{R} 57 \mathrm{~B} / 4$ I02B & $0.66 \pm 0.07$ & 0.54 & $0.62 \pm 0.04$ & 0.63 \\
\hline $9-6 / 14-2$ & $3 \mathrm{~L} 75 \mathrm{C} / 4$ 102B & $1.24 \pm 0.13$ & 0.99 & $1.29 \pm 0.11$ & 1.03 \\
\hline
\end{tabular}

CAT activity of strains carrying two hemizygous copies of the LTR-CAT construct (see Additional file I, Cross I for details of strain construction) are approximately equal to the values expected under the additive model (i.e., the sum of the CAT activities measured in two strains carrying single copies of the respective LTR-CAT constructs).

$\mathrm{X}$-chromosome in females, we selected rox as a putative positive control.

The results presented in Figure 5 indicate that all three proteins bind to copia elements in vivo (and to a lesser extent to rox) but presumably not within the context of the MSL complex since MSL-2 is not expressed in females

a

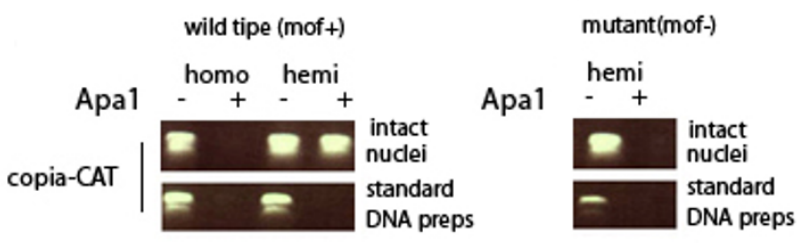

\section{Figure 2}

PCR amplification products (copia LTR-CAT primers shown in Figure I) of DNA prepared from intact nuclei (chromatin structure maintained) vs. DNA purified using standard procedures (chromatin structure not maintained-see Methods) digested (+) or not digested (-) with Apa I restriction enzyme. (a) The results indicate that the Apa I restriction site is accessible for digestion in nuclei preps (chromatin structure preserved) from wild-type larvae homozygous for the copia LTR-CAT construct but is not accessible for digestion in nuclei preps from larvae hemizygous for the construct in the wild-type genetic background. (b) The resistance of hemizygous copies of the copia LTR-CAT construct is lost in larvae homozygous for the mof' (loss-of-function) allele. All experiments were conducted with the 9-3 transformant strain. [wild type (mof $+)=$ DNA from larvae carrying the wild-type allele at the mof locus; mutant (mof - ) = DNA from larvae carrying the mof ${ }^{\prime}$ allele at the mof locus; homo = DNA isolated from larvae homozygous for the copiaLTR-CAT construct; hemi = DNA isolated from larvae hemizygous for the copiaLTR-CAT construct].
(Figure 5). Previous studies have demonstrated that the function of MOF within the context of the MSL complex is to acetylate lysine 16 on histone H4 (H4K16ac) [24], resulting in chromatin decondensation and increased transcription. In contrast, our results indicated that MOF is associated with reduced transcription of hemizygous copies of copia and perhaps other Drosophila LTR retrotransposons. Although the acetylation of histones is generally associated with transcriptional activation, it can also be involved in transcriptional repression [25-27]. Further studies will be required to determine the molecular basis of these findings.

\section{Discussion}

A determination of the mechanistic basis of MOF mediated silencing of copia remains to be determined. Nevertheless, the fact that hemizygous copies of copia and perhaps other families of Drosophila LTR retrotransposons are the target of epigenetic repression, appears to be analogous to MSUD (meiotic silencing by unpaired DNA) in Neurospora where DNA unpaired in meiosis causes silencing of the unpaired sequence and all DNA homologous to it $[28,29]$. Similar phenomena have been reported in $C$. elegans [30,31] and mice [32], and have been associated with epigenetically mediated modifications in chromatin structure. Our results indicate that the repression of hemizygous copies of copia and other Drosophila LTR retrotransposons is also mediated by changes in chromatin structure. The fact that this repression appears to be mediated by MOF implies a relationship between retrotransposons and the evolution of dosage compensation in Drosophila.

While it is generally acknowledged that MOF plays an essential role in equalizing the expression of X-linked and autosomal genes in Drosophila males, the mechanism(s) by which this is achieved remains controversial [33]. One model postulates that MOF, in association with other members of the MSL complex, binds to the hemizygous X- 
Table 4: Expression levels of copia LTR-CAT in stably transformed strains of Drosophila melanogaster hemizygous for the construct

\begin{tabular}{|c|c|c|c|c|}
\hline \multirow[t]{2}{*}{ GENE } & \multicolumn{2}{|c|}{ FEMALE } & \multicolumn{2}{|c|}{ MALE } \\
\hline & wild type & mutant & wild type & mutant \\
\hline Low & $0.85(0.08)$ & $0.73(0.10)$ & $0.62(0.05)$ & $0.64(0.12)$ \\
\hline HDAC I326 & $1.60(0.27)$ & $0.97(0.16)^{*}$ & $1.31(0.31)$ & $0.93(0.17)$ \\
\hline HDAC I328 & $"$ & $0.88(0.15)^{*}$ & " & $0.96(0.20)$ \\
\hline$E(z)^{61}(29 C)$ & $0.54(0.09)$ & $0.5 \mathrm{I}(0.09)$ & $0.38(0.09)$ & $0.43(0.09)$ \\
\hline " (25 C) & " & $0.42(0.08)$ & " & $0.38(0.10)$ \\
\hline " (I8 C) & $"$ & $0.4 \mid(0.07)$ & " & $0.42(0.12)$ \\
\hline$E(z)^{28}(29 C)$ & $"$ & $0.34(0.07)^{*}$ & $"$ & $0.34(0.07)$ \\
\hline$"(25 \mathrm{C})$ & $"$ & $0.40(0.12)$ & $"$ & $0.33(0.10)$ \\
\hline " (I8C) & $"$ & $0.28(0.06)^{*}$ & $"$ & $0.29(0.08)$ \\
\hline$P_{s c^{25}}$ & " & $0.17(0.03)^{*}$ & " & $0.23(0.03)^{*}$ \\
\hline$S x \|^{f}$ & $0.39(0.26)$ & $0.55(0.12)$ & $0.83(0.12)$ & $0.79(0.14)$ \\
\hline mof ${ }^{\prime}$ & NA & NA & $0.79(0.07)$ & $3.21(0.35)^{* *}$ \\
\hline
\end{tabular}

copia LTR-CAT in a transformed strain (9-3) made wild type or heterozygous for dominant mutant alleles of genes known to affect chromatin structure [LOW-lightening of white; HDAC 1326, HDAC 1328. Histone Deacetylase; $E(z)^{61}, E(z)^{28-E n h a n c e r ~ o f ~ z e s t e ; ~ P s c ~}{ }^{25}$-Posterior sex combs; Sx ff-Sex lethal or hemizygous for the X-linked recessive mof ${ }^{\prime}$ (males absent on first) allele. A slight but significant decrease in activity was observed in strains heterozygous for the dominant mutant HDAC 1326, HDAC 1 ${ }^{328}, E(z)^{28}$ and Psc ${ }^{25}$ alleles. A highly significant increase in activity was observed in the strain hemizygous for the recessive mutant mof' allele

chromosome resulting in a two-fold increase in X-linked gene expression [34,35]. According to this model, MOF acetylates $\mathrm{H} 4$ on lysine 16 [H4Ac16] resulting in a relaxed chromatin configuration and a consequent elevation in gene expression. While our data indicate that MOF interacts with hemizygous copies of copia, the consequence is repression rather than elevation in expression. This suggests that the regulatory functions of Drosophila MOF may be context dependent.

The alternative "inverse regulator model" of dosage compensation postulates that hemizygosity of the male Xchromosome results is a genome-wide elevation in gene expression $[19,36]$. The significance of the sequestering of the MSL complex to the male X-chromosome in this model is two-fold. First removal of the MOF acetyltransferase from autosomes is postulated to attenuate the increased level of autosomal gene expression due to the $\mathrm{X}$ dosage affect. Secondly, the binding of the MSL complex prevents X-linked genes from responding to the elevated levels H4Ac16. The mechanism(s) underlying this second proposed function is unknown, although it has been demonstrated that the expression of at least some Xlinked and autosomal genes are significantly elevated in male mof mutants $[19,36]$. This suggests that MOF alone or within the context of the MSL complex can act to repress expression of at least some X-linked genes. This finding is consistent with our observation that MOF represses copia expression in the hemizygous condition. a

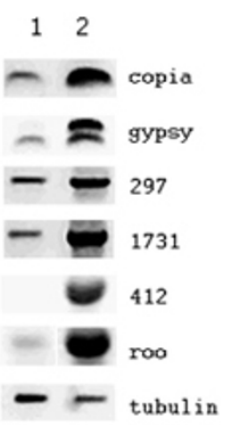

b

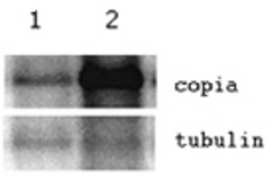

Figure 3

(a). RT-PCR of mRNA isolated from 3rd instar larvae wild-type (column I) or mutant (mof') (column 2) at the mof locus using primers specific for five Drosophila melanogaster LTR retrotransposons (copia, gypsy, 297, I73I, 4I 2 and roo) and $\beta$-tubulin as a control. The results demonstrate uniformly higher levels of LTR retrotransposon expression in larvae carrying the mutant mof' allele (see Methods for primer sequences). (b). Northern hybridization of mRNA isolated from 3rd instar larvae wild-type (column I) or mutant (mof') (column 2) at the mof locus using copia [43] and $\beta$-tubulin [44] probes. The results are consistent with RT PCR analyses (Figure 3a) and demonstrate higher levels of copia expression in flies carrying the mutant mof ${ }^{\prime}$ allele. No significant difference in $\beta$-tubulin expression was detected.

Since retrotransposons significantly predate the evolution of dosage compensation, our results suggest that the original function of MOF and perhaps other members of the MSL complex was to silence retrotransposons and that these functions were later co-opted in the evolution of dosage compensation in Drosophila.

\section{Conclusion}

A growing body of evidence in both plants and animals indicates that epigenetic mechanisms originally evolved as a defense against transposable elements and were subsequently co-opted for a variety of cellular functions [3739]. Our findings are consistent with this model and suggest that at least some of the mechanisms underlying dosage compensation in Drosophila may have their origins in processes originally evolved to defend against the mutagenic potential of transposable elements.

\section{Methods}

\section{Fly strains}

All mutant fly strains were obtained from the Bloomington Stock Center. Wild strain 194 was established from a collection in Athens (Georgia, USA) in June 2003. Crosses were performed at $25^{\circ} \mathrm{C}$ on yeast, cornmeal, molasses, and agar medium. Details of the Drosophila strains and genetic crosses used in the construction of flies/larvae used in this study are provided in the Additional file 1. 
a

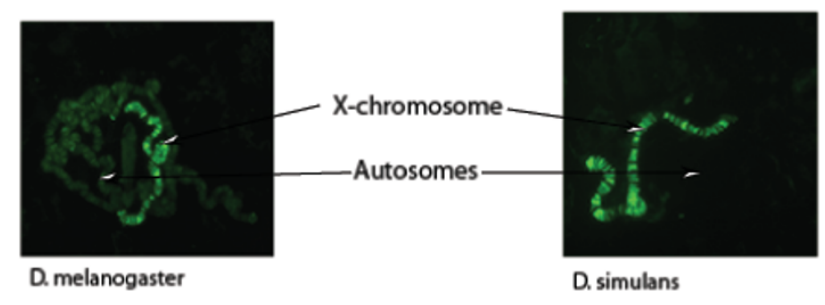

Figure 4

Immunostaining of Drosophila polytene chromosomes with MOF-antibody. Shown are preparations from strains representing two Drosophila species- (a) D. melanogaster and (b) D. simulans. Reduced binding of MOF to D. simulans autosomes is consistent with the reduced number of LTR retrotransposons present in this species.

\section{Transgenic flies and CAT-assay}

The full-length copia LTR-CAT construct described previously [40] was sub-cloned from $p$ CopiaCAT into Pst1-Xba1 site of the $p C a S p e R$ [41] transformation vector. Germ line transformation was performed by microinjection of $1 \mathrm{~h}$ embryos. Injected survivors were backcrossed to $w^{1118}$ males or females and transformants were identified by eye color. Insertion sites were determined by in situ hybridization of pCAT plasmid (Promega) to polytene salivary gland chromosomes of third instar larvae. The probe was labeled with biotin (bio-dUTP) by nick-translation. Hybridization was detected using the Vestastain ABC kit (Vector Labs) and diaminobenzidine (Sigma). The transgene locations were determined according to standard maps of Lefevre [42].

CAT activity was measured by liquid scintillation counting (LSC) of CAT reaction products. Crude protein extract from 1 fly/larva was incubated in a reaction mix containing C-14 chloramphenicol and n-butyryl Coenzyme A (Promega). Eight to10 flies were assayed individually for each variant (strain). Results are presented in CAT units per fly. 1 CAT unit is defined as the conversion of $1 \mathrm{nmol}$ acetyl coenzyme A to chloramphenicol/min at $37^{\circ} \mathrm{C}$. Activity values are the average of 10 independent fly or larvae assays per strain. Means and standard deviations of CAT activity units were computed for each strain. Two tailed t-tests were used to test the significance.

\section{Northern analysis and RT-PCR}

mRNA was isolated from 3rd instar larvae using the Oligotex Direct mRNA kit (Qiagen, Valencia, CA). RNA was electrophoresed through a 1\% agarose-formaldehyde gel, transferred to nitrocellulose filter and hybridized as described [43]. The copia genomic clone DM5002 [43] was used as a probe. For loading control a $\beta$-tubulin probe

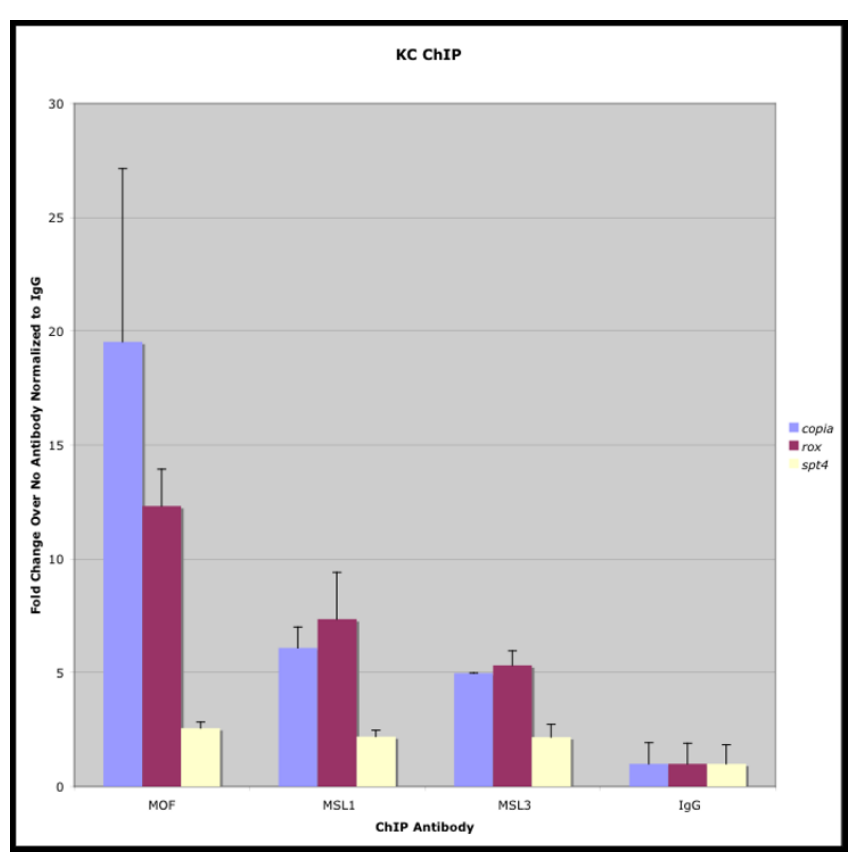

Figure 5

MOF is physically associated with the copia untranslated leader region (ULR) in vivo. Chromatin immunoprecipitation (ChIP) experiments were performed to determine whether MOF is physically associated with copia DNA sequences in vivo. Precipitation reactions performed independently with the MOF, the MSL-I and MSL-3 antibodies, rabbit IgG antibody (non-specific control) and no antibody were used as templates for quantitative PCR from Kc cells. The average and standard errors for triplicate reactions were plotted to reveal the average fold increase in precipitated copia DNA for each antibody relative to the no antibody reactions. Precipitated DNA was amplified using primers specific for the copia ULR, the roX-I gene (positive control) and the Spt4 gene (negative control) (see Methods). MOF and other members of the MSL complex have previously been demonstrated to bind to the roX-I gene [e.g., 23]. Spt-4 was chosen as a negative control because it is not a target site for the MSL complex [e.g., 22]. The results indicate that MOF, MSL-I and MSL-3 are all associated with the copia ULR and roX-I in vivo. There was no binding above background to the Spt-4 gene.

[44] was included into the hybridization mix. Probes were labeled using a nick-translation kit (Roche).

For RT-PCR, mRNA was additionally treated with DNase (DNA-free kit, Ambion). cDNA was synthesized with Oligo(dT) primers, ThermoScript RT-PCR System (Invitrogen). PCR primers: roo-f 5'- TCC ATT CAA GGA TGT CAC C-3'; roo-r 5'- ATG CTT TTT CGG AGG CGT CC-3'; 1731-f 5'-GCC ATT TGA ATA CAA GCA GCC TAC-3'; 1731-r 5'- CGG GAT TAG CAG CAT CTG TGA AC-3'; 412f 5'- CAG TGT GCT AAG GCT TTG AAC CTA c-3'; 412-r 5'GAA CTT GGG CTT GTA TTT CTT CCA C-3'; 297-f 5'- ATT 
Table 5: Expression levels of copia LTR-CAT in stably transformed strains of Drosophila melanogaster hemizygous for the construct

\begin{tabular}{cll}
\hline STRAIN & mof + hemizygous copia LTR-CAT & mof - hemizygous copia LTR-CAT \\
\hline $9-3$ & $0.79(0.07)$ & $3.21(0.35)^{* *}$ \\
$9-4$ & $0.31(0.05)$ & $2.53(0.61)^{* *}$ \\
$9-6$ & $0.72(0.10)$ & $3.97(0.21)^{* *}$ \\
$14-2$ & $0.28(0.08)$ & $1.35(0.27)^{* *}$
\end{tabular}

Expression levels of copia LTR-CAT in males made hemizygous for the construct in a series of transformed strains (copia LTR-CAT inserted at different chromosomal locations in each strain-see Table I) in wild-type vs. mof' genetic backgrounds. The results indicate a position independent repression of hemizygous copies of the copia LTR-CAT by the wild-type mof allele ( ${ }^{*} \mathrm{p}<0.01$; $\left.{ }^{* *} \mathrm{p}<0.00 \mathrm{I}\right)$.

GCC AGT GAC CAT CAA CCT C-3'; 297-r 5'- TGC TAC CCC GTT TTT TGC TG-3'; copia-f 5'-GGG AAG AAG CCA TCA ATA CAG-3'; copia-r 5'-CAA ATA CTT CAA ACC AGC ATC-3'; gypsy-f 5'- CGT AAT AAG TGT GCG TTG AAT-3'; sypsy-r 5'- CGA CCT TAA CCT TTC TGT AGT-3'; $\beta$-tubulinf 5'- CAA GGC TTC CAA CTC ACA CAC TC-3'; $\beta$-tubulinr 5'- AGG TGG CGG ACA TCT TCA GAC-3'.

\section{Nuclear isolation and chromatin analyses}

Nuclei were prepared from third instar larvae, such that chromatin structure is preserved as described previously [12]. Standard DNA extraction (chromatin structure not preserved) from the same stage larvae was carried out using proteinase $\mathrm{K}$ digestion overnight followed by phenol-chloroform extraction. Digestion of nuclei or DNA with Apa 1 was performed at $37^{\circ} \mathrm{C}$ for $1 \mathrm{~h}$. Reactions were stopped by heating at $70^{\circ} \mathrm{C}$ for $10 \mathrm{~min}$. PCR were performed with the following primers: copia-f 5'-GGG AAG AAG CCA TCA ATA CAG-3' copia-r 5'-CAA ATA CTT CAA ACC AGC ATC-3'; CAT-r 5'-CAC CGT CTT TCA TTG CCA TAC G 3'(See Figure 1).

\section{Chromatin immunoprecipitation}

Chromatin immunoprecipitation was performed on the Drosophila melanogaster Kc167 cell line (female, by criterion of dsx splicing) obtained from the Drosophila Genomics Resource Center. Cells were grown to a density of $2 \times$ $10^{6}$ to $4 \times 10^{6}$ cells $/ \mathrm{ml}$. EZ CHIPTM Chromatin Immunoprecipitation Kit from Upstate Inc. (Chicago) was used according to manufactures instructions. MOF, MSL- 1 and MSL-3 antibodies [21] were provided by Dr. John Lucchesi (Emory University), and normal mouse IgG antibody (Upstate Inc., Chicago) was used as a nonspecific control.

The chromatin immunoprecipitation polymerase chain reactions were quantified using the $2^{-\Delta \Delta \mathrm{C} T}$ method [45]. PCRs were performed with primers specific for the copia ULR ( 5 ' untranslated leader region; f 5'GCCCAGTCCATGCCTAATAA-3'; r 5'-GCCTTGTCCATTTTTCACTCA-3'), the roX-1 gene [23] (positive control; f 5'-GTCGAATTCGAAAAACACATTTACTAACAAATAA-3'; r 5'-GTCGAATTCCCCAAAGAAATCCACATAACAT-3') and the Spt-4 gene [22] (negative control; f 5'-CTCGTGGTATCTATGCCATTTCTG-3'; r 5'-TCCACGATTCTTCATGTCACGTA-3') in the presence of cyber green. Reactions were monitored on the DNA Engine Opticon 2 Continuous Fluorescence Detector. The Opticon Monitor 2 Software v2.01 was used to calculate the $\mathrm{C}_{\mathrm{T}}$ for each reaction following subtraction of the minimum over cycle range background and manually setting the threshold to the linear range of amplification. Triplicate polymerase chain reactions were performed for each antibody precipitation. The average of three polymerase chain reactions of no antibody (beads only) precipitations was subtracted from each polymerase chain reaction with antibody to generate three $\Delta \mathrm{C}_{\mathrm{T}}$ values for each immunoprecipitation. The average of three polymerase chain reactions of the IgG immunoprecipitation (nonspecific control) was subtracted from each $\Delta \mathrm{C}_{\mathrm{T}}$ value to yield three $\Delta \Delta \mathrm{C}_{\mathrm{T}}$ values for each immunoprecipitation normalized to IgG. The average and standard errors for the three $2^{-\Delta \Delta \mathrm{C} T}$ values were plotted to reveal the average fold increase of antibody precipitation reactions over the no antibody precipitation reaction controls.

\section{Authors' contributions}

LVM helped conceive the study, conducted the experiments and contributed to the data analysis; NJB contributed to the data analysis; JFMcD conceived the study, contributed to the data analysis and wrote the paper.

\section{Additional material}

\section{Additional file 1}

Drosophila strains and genetic crosses. Drosophila strains and genetic crosses used in the construction of flies/larvae used in this study Click here for file

[http://www.biomedcentral.com/content/supplementary/14712199-9-55-S1.doc]

\section{Additional file 2}

Table 1-5. CAT activity in larvae or adult flies hemizygous for the construct within a series of genetic backgrounds mutant for genes known to affect chromatin structure in Drosophila:LOW/+, HDAC 1326/+, HDAC $1328 /+, E(z)^{28} /+, E(z)^{61 /+}$, Psc $25 /+$, Sxl ${ }^{\mathrm{fl}} /+$, presence of extra $Y$ chromosome or absence of $Y$ chromosome.

Click here for file

[http://www.biomedcentral.com/content/supplementary/14712199-9-55-S2.doc] 


\section{Acknowledgements}

We are grateful to Dr. John C. Lucchesi (Emory University) for providing MOF and MSL antibodies and to Dr. DeEtte Walker for editorial assistance.

This research was supported by an NIH grant to JM.

\section{References}

I. Coffin JM, Hughes JM, Varmus HE: Retroviruses Plainview: Cold Spring Harbor Press; 1997

2. Matzke MA, Mette MF, Matzke AJ: Transgene silencing by the host genome defense: implications for the evolution of epigenetic control mechanisms in plants and vertebrates. Plant Mol Biol 2000, 43:40I-4I5.

3. Bestor TH: DNA methylation: evolution of a bacterial immune function into a regulator of gene expression and genome structure in higher eukaryotes. Philos Trans $R$ Soc Lond B Biol Sci 1990, 326: I79-187.

4. Barlow DP: Methylation and imprinting: from host defense to gene regulation? Science 1993, 260:309-310.

5. Ganko EW, Greene CS, Lewis JA, Bhattacharjee V, McDonald JF: LTR retrotransposon gene associations in Drosophila melanogaster. I Mol Evol 2006, 62: I I I-120.

6. Bradshaw GJ, Baker BS: Dosage compensation and chromatin structure in Drosophila. Curr Opin Genet Dev 1996, 6:496-50I.

7. Pirrotta V: Polycomb silencing mechanisms and genomic programming. Ernst Schering Res Found Workshop 2006, 57:97-I I3.

8. Lyko F, Beisel C, Marhold J, Paro R: Epigenetic regulation in Drosophila. Curr Top Microbiol Immunol 2006, 31 0:23-44.

9. Schulz WA, Steinhoff C, Flori AR: Methylation of endogenous human retroelements in health and disease. Curr Top Microbiol Immunol 2006, 3 1 0:2 I I-250.

10. Huettel B, Kanno T, Daxinger L, Aufsatz W, Matzke J, Matzke M: Endogenous targets of RNA-directed DNA methylation and Pol IV in Arabidopsis. EMBO J 2006, 25:2828-2836.

II. Kavi HH, Fernandez HR, Birchler JA: RNA silencing in Drosophila. FEBS Lett 2005, 579:5940-5949.

12. Cartwright IL, Cryderman DE, Gilmour DS, Pile LA, Wallrath LL, Weber JA, Elgin SC: Analysis of Drosophila chromatin structure in vivo. Methods Enzymol 1999, 304:462-496.

13. Simon JA, Tamkun JW: Programming off and on states in chromatin: mechanisms of Polycomb and trithorax group complexes. Curr Opin Genet Dev 2002, 12:210-218.

14. Birchler JA, Bhadra U, Bhadra MP, Auger DL: Dosage-dependent gene regulation in multicellular eukaryotes: implications for dosage compensation, aneuploid syndromes, and quantitative traits. Dev Biol 200I, 234:275-288.

15. Hilfiker A, Hilfiker-Kleiner D, Pannuti A, Lucchesi JC: Mof, a putative acetyl transferase gene related to the Tip60 and MOZ human genes and to the SAS genesof yeast, is required for dosage compensation in Drosophila. EMBO J 1997, 1 6:2054-2060.

16. Buscaino A, Kocher T, Kind JH, Holz H, Taipale M, Wagner K, Wilm M, Akhtar A: MOF-regulated acetylation of MSL-3 in the Drosophila dosag compensation complex. Mol Cell 2003, I I: |265-I277.

17. Bhadra MP, Bhadra U, Kundu J, Birchler JA: Gene expression analysis of the function of the male-specific lethal complex in Drosophila. Genetics 2005, 169:206|-2074.

18. Morales V, Straub T, Neumann MF, Mengus G, Akhtar A, Becker PB: Functional integration of the histone acetyltransferase $M O F$ into the dosage compensation complex. EMBO J 2004, 23:2258-2268.

19. Bhadra U, Pal-Bhadra M, Birchler JA: Role of the male specific lethal (msl) genes in modifying the effects of sex chromosomal dosage in Drosophila. Genetics 1999, I 52:249-68.

20. Vieira C, Biemont C: Transposable element dynamics in two sibling species: Drosophila melanogaster and Drosophila simulans. Genetica 2004, I 20: I I5-123.

21. Gu W, Wei X, Pannuti A, Lucchesi JC: Targeting the chromatinremodeling MSL complex of Drosophila to its sites of action on the $X$ chromosome requires both acetyl transferase and ATPase activities. EMBO J 2000, 19:5202-52II.

22. Furuhashi H, Nakajima M, Hirose $S$ : DNA supercoiling factor contributes to dosage compensation in Drosophila. Development 2006, I 33:4475-4483.
23. Henry RA, Tews B, Li X, Scott MJ: Recruitment of the male-specific lethal (MSL) dosage compensation complex to an autonomously integrated roX chromatin entry site correlates with an increased expression of an adjacent reporter gene in male Drosophila. J Biol Chem 200I, 276:31953-31958.

24. Smith ER, Pannuti A, Gu W, Steurnagel A, Cook RG, Allis CD, Lucchesi JC: The Drosophila MSL complex acetylates histone H4 at lysine 16, a chromatin modification linked to dosage compensation. Mol Cell Biol 2000, 20:312-318.

25. Eberharter A, Becker PB: Histone acetylation: a switch between repressive and permissive chromatin. EMBO Rep 2002, 3:224-229.

26. Kurdistani SK, Tavazoie S, Grunstein M: Mapping global histone acetylation patterns to gene expression. Cell 2004 I I 7:721-733

27. Rea S, Xouri G, Akhtar A: Males absent on the first (MOF): from flies to humans. Oncogene 2007, 26:5385-5394.

28. Shiu PK, Metzenberg RL: Meiotic silencing by unpaired DNA: properties, regulation and suppression.

29. Nakayashiki $H$ : RNA silencing in fungi: Mechanisms and applications. FEBS Lett 2005, 579:5950-5957.

30. Bean CJ, Schaner CE, Kelly WG: Meiotic pairing and imprinted $\mathbf{X}$ chromatin assembly in Caenorhabditis elegans. Nat Genet 2004, 36:100-105.

3I. Maine EM, Hauth J, Ratliff T, Vought V, She X, Kelly WG: EGO-I, a putative RNA-dependent RNA polymerase, is required for heterochromatin assembly on unpaired DNA during $C$. elegans meiosis. Curr Biol 2005, I 5: I972-1978.

32. Turner JM, Mahadevaiah SK, Fernandez-Capetillo O, Nussenzwieg A, $\mathrm{Xu}$ X, Deng CX, Burgoyne PS: Silencing of unsynapsed meiotic chromosomes in the mouse. Nat Genet 2005, 37:41-47.

33. Larsson J, Meller VH: Dosage compensation, the origin and the afterlife of sex chromosomes. Chrom Res 2006, I4:4I7-43I.

34. Hamada FN, Park PJ, Gordadzw PR, Kuroda MI: Global regulation of $\mathrm{X}$ chromosomal genes by the MSL complex in Drosophila melanogaster. Genes Dev 2005, 19:2289-2294.

35. Straub T, Gilfillan GD, Maier VK, Becker PB: The Drosophila MSL complex activates the transcription of target genes. Genes Dev 2005, 1 9:2284-2288.

36. Birchler JA, Pal-Bhadra M, Bhadra U: Dosage dependent gene regulation and the compensation of the $X$ chromosome in Drosophila males. Genetica 2003, I I 7: I79-190.

37. Henikoff S, Matzke MA: Exploring and explaining epigenetic effects. Trends Genet I997, I3:293-295

38. McDonald JF, Matzke MA, Matzke AJ: Host defenses to transposable elements and the evolution of genomic imprinting. Cytogenet Genome Res 2005, I 1 0:242-249.

39. Slotkin RK, Martienssen R: Transposable elements and the epigenetic regulation of the genome. Nat Rev Genet 2007, 8:272-285.

40. Matyunina LV, Jordan IK, McDonald JF: Naturally occurring variation in copia expression is due to both element (cis) and host (trans) regulatory variation. Proc Natl Acad Sci USA 1996, 93:7097-7102.

41. Rubin GM, Spradling AC: Vectors for $\mathbf{P}$ element-mediated gene transfer in Drosophila. Nucleic Acids Res 1983, I I:6341-635I.

42. Lindsley DL, Zimm GG: The Genome of Drosophila melanogaster San Diego: Academic Press; 1992

43. Csink AK, McDonald JF: Copia expression is variable among natural populations of Drosophila. Genetics 1990, I 26:375-85.

44. Bialojan S, Falkenburg D, Renkawitz-Pohl R: Characterization and developmental expression of beta tubulin genes in Drosophila melanogaster. EMBO J 1984, 3:2543-2548.

45. Livak KJ, Schmittgen TD: Analysis of relative gene expression data using real-time quantitative PCR and the 2(-Delta Delta C(T)) method. Methods 200I, 25:402-408. 